\title{
A Study on Impaired Awareness of Hypoglycaemia in Type 1 Diabetes Patients in a Tertiary Care Centre in Singapore
}

\author{
Marvin Chua ${ }^{1}$, Ester Yeoh $^{2}$, Sharon Fun ${ }^{3}$, and Angela Koh ${ }^{1}$ \\ ${ }^{1}$ Sengkang General Hospital \\ ${ }^{2}$ Admiralty Medical Centre \\ ${ }^{3}$ Khoo Teck Puat Hospital
}

April 27, 2021

\begin{abstract}
Background: This study aims to establish the frequency of severe hypoglycaemia and impaired awareness of hypoglycaemia (IAH) in Type 1 diabetes (T1D) patients in Singapore. We evaluate the utility of the Gold and Clarke scores as screening tools for IAH, and assess the effects of diabetes self-management practices and education in reducing IAH. Methods: This is an observational study of 131 subjects with T1D at a regional hospital, which included review of medical records, self-administered questionnaires and a prospective 4 week period of self-monitoring of blood glucose (SMBG). Results: The frequency of clinically significant hypoglycaemia (blood glucose $<3 \mathrm{mmol} / \mathrm{L}$ ) during the 4 week period of SMBG was $38.8 \%$, while the frequency of severe hypoglycaemia over the last one year was $22.5 \%$. Based on the Gold score and Clarke score, $28.3 \%$ and $24.6 \%$ of subjects respectively had IAH. The Clarke score was associated with increased frequency of clinically significant hypoglycaemia, severe hypoglycaemia and hospitalizations for hypoglycaemia. Prior group education, education from a dietitian and education on blood glucose targets were associated with lower Clarke scores, while adjusting insulin doses for blood glucose using an insulin sensitivity factor was associated with lower Gold and Clarke scores. Conclusion: IAH is common in T1D patients in Singapore and is associated with increased risk of severe hypoglycaemia. The Gold and Clarke scores are simple tools which can be routinely administered to identify patients with IAH who might benefit from specific interventions, particularly structured diabetes education, to decrease IAH and its complications.
\end{abstract}

\section{TITLE}

A Study on Impaired Awareness of Hypoglycaemia in Type 1 Diabetes Patients in a Tertiary Care Centre in Singapore

\section{ABSTRACT}

Background:

This study aims to establish the frequency of severe hypoglycaemia and impaired awareness of hypoglycaemia (IAH) in Type 1 diabetes (T1D) patients in Singapore. We evaluate the utility of the Gold and Clarke scores as screening tools for IAH, and assess the effects of diabetes self-management practices and education in reducing IAH.

Methods:

This is an observational study of 131 subjects with T1D at a regional hospital, which included review of medical records, self-administered questionnaires and a prospective 4 week period of self-monitoring of blood glucose (SMBG).

Results: 
The frequency of clinically significant hypoglycaemia (blood glucose $<3 \mathrm{mmol} / \mathrm{L}$ ) during the 4 week period of SMBG was $38.8 \%$, while the frequency of severe hypoglycaemia over the last one year was $22.5 \%$. Based on the Gold score and Clarke score, $28.3 \%$ and $24.6 \%$ of subjects respectively had IAH. The Clarke score was associated with increased frequency of clinically significant hypoglycaemia, severe hypoglycaemia and hospitalizations for hypoglycaemia. Prior group education, education from a dietitian and education on blood glucose targets were associated with lower Clarke scores, while adjusting insulin doses for blood glucose using an insulin sensitivity factor was associated with lower Gold and Clarke scores.

Conclusion:

IAH is common in T1D patients in Singapore and is associated with increased risk of severe hypoglycaemia. The Gold and Clarke scores are simple tools which can be routinely administered to identify patients with IAH who might benefit from specific interventions, particularly structured diabetes education, to decrease IAH and its complications.

Keywords: hypoglycaemia, impaired awareness of hypoglycaemia, severe hypoglycaemia, Type 1 Diabetes

\section{What is already known about this topic?}

Impaired awareness of hypoglycaemia (IAH) is common in people with Type 1 diabetes (T1D) and is an important clinical problem which leads to increased risk of severe hypoglycaemia, increased morbidity and mortality and decreased quality of life. Despite this, objective methods to screen for IAH are not in routine clinical use. Education plays a central role in the potential reduction of IAH.

\section{What does this article add?}

This is the first study on IAH in T1D patients in Singapore, with no prior reported studies in the literature. Most of the prior studies on IAH have been performed in Caucasian, rather than Asian populations. Thus, our study would provide useful information for clinicians practising in Asian countries where Diabetes Mellitus is a burgeoning problem.

Our study illustrates the utility of the Gold and Clarke scores, simple clinical tools which can be administered in an outpatient setting, to screen for IAH in patients with T1D. We assess the association of both scores with the important clinical outcome of severe hypoglycaemia, and compare the performance of both scores with each other. Our study is the first to compare the performance of both the Gold and Clarke scores with the HYPO score - a composite hypoglycaemia score based on the frequency, severity and degree of unawareness of hypoglycaemia. As calculation of the HYPO score is highly laborious and difficult to do in routine clinical practice, our findings confirm that a simple questionnaire-based tool is comparable with a far more rigorous method for assessment of hypoglycaemia.

Our study is also novel in that it provides an analysis of the specific content and delivery of education which is most efficacious in reducing IAH in patients with T1D. For example, group education, education on self-adjustment of insulin doses and blood glucose targets are particularly useful.

In summary, our study provides several clinical pearls of practical significance which would be useful for all clinicians managing patients with T1D.

\section{INTRODUCTION}

The Diabetes Control and Complications trial (DCCT) has clearly demonstrated that in people with Type 1 Diabetes (T1D), good glycaemic control leads to decreased risk of diabetes-related complications ${ }^{1}$. However, hypoglycaemia and impaired awareness of hypoglycaemia (IAH) are important barriers to optimization of glycaemic control.

Hypoglycaemia is defined as an episode of abnormally low plasma glucose that exposes the individual to potential harm ${ }^{2}$. Hypoglycaemia can be classified into three levels: a hypoglycaemia alert value (glucose $=<$ $3.9 \mathrm{mmol} / \mathrm{L}$ ), clinically significant hypoglycaemia (glucose $<3 \mathrm{mmol} / \mathrm{L}$ ) and severe hypoglycaemia, which 
is hypoglycaemia associated with severe cognitive impairment requiring external assistance for recovery ${ }^{3}$. Impaired awareness of hypoglycaemia (IAH) is defined as a diminished ability to perceive the onset of hypoglycaemia ${ }^{4}$. IAH is an important clinical problem in people with T1D which leads to increased risk of severe hypoglycaemia (SH), increased morbidity and mortality and decreased quality of life ${ }^{5-9}$.

Despite this, there have been no prior studies on IAH done in T1D patients in Singapore. We aimed to assess the prevalence of IAH in Singapore, including its frequency, impact and association with diabetes self-management practices, in T1D patients. We also aimed to evaluate the use of the Gold and Clarke scores, both of which can be routinely administered in clinical practice, for assessment of IAH.

\section{METHODS}

All patients with T1D who were seen at the Diabetes Centre of a regional hospital were invited to participate in this study. Exclusion criteria included patients who were pregnant, English illiterate or had significant cognitive impairment that negated taking an informed consent. Informed consent was obtained from subjects who agreed to participate in the study, with ethics approval obtained from the National Healthcare Group Domain Specific Review Board. This study consisted of 3 components: review of medical records, a detailed self-administered questionnaire (in English) and review of the blood glucose profile based on prospectively performed self-monitoring of blood glucose (SMBG) for 4 weeks.

Review of medical records included demographic data such as the patient's age, gender and ethnicity, body mass index (BMI), age of diabetes diagnosis and the duration of diabetes, HbA1c values, results of insulin autoantibodies, as well as the insulin type and doses. The presence of diabetes-related complications both microvascular complications such as retinopathy, nephropathy, neuropathy and macrovascular complications such as ischemic heart disease, stroke and peripheral vascular disease; were included. All prior hospitalizations or emergency department attendances for hypoglycaemia were also reviewed.

The questionnaire included several categories of important information. Firstly, diabetes self-management practices were assessed, which included frequency of SMBG (at least 3 times/day), methods for dosing of insulin (fixed or flexible insulin dosing), methods used to adjust insulin doses (based on carbohydrate intake and/or blood glucose), familiarity with carbohydrate counting and correction for hyperglycaemia. Secondly, prior diabetes education was assessed including the format, delivery and content covered. Thirdly, the Gold score and Clarke score, which are screening tools for $\mathrm{IAH}^{6,8,10}$, were assessed. The Gold score was obtained using a single question "do you know when your hypoglycaemic episodes are commencing?", with the response graded on a 7-point Likert scale: 1 representing "always aware" and 7 representing "never aware" ${ }^{6}$. A Gold score of $>=4$ indicates IAH ${ }^{6}$. The Clarke score comprises eight questions assessing the exposure to hypoglycaemia, symptomatic responses to hypoglycaemia and the glycaemic threshold ${ }^{8}$. A Clarke score of $>=4$ indicates IAH ${ }^{8}$. Last but not least, the Problem Areas in Diabetes (PAID) questionnaire, a 20-item scale consisting of emotional problems commonly seen in people with diabetes mellitus, was administered.

The HYPO score, a useful indicator of the severity of problems with hypoglycaemia, was computed ${ }^{11}$. This score comprised of two components - the first component was based on historical recall of hypoglycaemic events requiring assistance during the preceding one year - assistance required to recognize hypoglycaemia, assistance required to treat hypoglycaemia, glucagon administration required for hypoglycaemia, and emergency medical services required for hypoglycaemia, in the last one year ${ }^{11}$. Severe hypoglycaemia was defined in our study as the occurrence of at least one of the above four events in the preceding one year. This is consistent with the American Diabetes Association definition of severe hypoglycaemia as severe cognitive impairment requiring external assistance for recovery ${ }^{2}$. The accuracy of self-recall of severe hypoglycaemia was confirmed by Pedersen et al, who demonstrated that $90 \%$ of subjects with confirmed episodes of severe hypoglycaemia during a prospective one year period of monitoring were able to successfully recall these episodes ${ }^{12}$. The second component of the HYPO score was calculated based on the prospective period of SMBG, as discussed in the next section.

Following administration of the questionnaire, subjects who consented were provided with a standardized glucometer and 150 test strips and instructed to perform SMBG before every meal and at bedtime, daily for 
4 weeks. Clinically significant hypoglycaemia was defined as an episode in which capillary blood glucose was $<3 \mathrm{mmol} / \mathrm{L}$ during this prospective 4 -week period of SMBG. A glucose level of $<3 \mathrm{mmol} / \mathrm{L}$ was chosen as it is sufficiently low to indicate serious, clinically important hypoglycaemia ${ }^{13}$. During all episodes in which blood glucose was $<3 \mathrm{mmol} / \mathrm{L}$, subjects were asked to record the absence or presence of symptoms and whether these were autonomic or neuroglycopenic symptoms - this was the second component required for calculation of the HYPO score.

Statistical analysis was performed using the Statistical Package for the Social Sciences (SPSS) software. Descriptive statistics were presented as median with interquartile range for quantitative variables and number (percentages) for qualitative variables. Univariate analysis was performed by using independent sample ttest, Pearson's chi-square test, Mann-Whitney-U test and Fisher exact test whenever appropriate to compare demographic and clinical factors between subjects with impaired and intact awareness of hypoglycaemia. A p-value of $<0.05$ was considered statistically significant and all p-values reported were two-sided.

\section{RESULTS}

A total of 131 subjects with T1D were recruited. Of the 151 subjects screened for enrolment, 1 demised, 2 had cognitive impairment, 14 were English illiterate and 3 declined. The baseline characteristics of the subjects are shown in Table 1 .

\section{Demographics}

Forty-eight percent of subjects were male; $72.5 \%$ were Chinese, $10.7 \%$ were Malay, $12.2 \%$ were Indian and $4.6 \%$ belonged to other ethnic groups.

Diabetes duration, complications, treatment and self-management practices

The median age of diabetes diagnosis was 19 (12 - 27) years, while the median duration of diabetes was 14 $(8-19)$ years. Median HbA1c was $8.2(7.3-9.2) \%$. Of the subjects, $15.3 \%$ had nephropathy as evidenced by microalbuminuria $(30-299 \mathrm{mg} / \mathrm{g}$ creatinine) or macroalbuminuria ( $>=300 \mathrm{mg} / \mathrm{g}$ creatinine), $6.9 \%$ had neuropathy and $15.3 \%$ had retinopathy. Macrovascular complications were present only in a minority; $3.8 \%$ had ischemic heart disease, $0.8 \%$ had stroke and $0.8 \%$ had peripheral vascular disease. Glutamic acid decarboxylase (GAD) antibody results were available for $61.8 \%$ of subjects, of which $76.5 \%$ were positive. Islet-cell antibody results were available for $24.4 \%$ of subjects, of which $21.9 \%$ were positive. Almost all (94\%) subjects were on multiple daily injections of insulin, as defined by 3 injections or more per day; $90 \%$ were using insulin analogs, with $83 \%$ on long acting basal analogs and $86 \%$ on rapid-acting analogs. The median insulin dose was $0.77(0.61-0.91)$ units $/ \mathrm{kg} /$ day.

The majority (93.9\%) of subjects were on flexible insulin dosing, with $91.1 \%$ adjusting insulin doses for food and $87.0 \%$ adjusting insulin doses for glucose. However, only $49 \%$ of subjects reported familiarity with carbohydrate counting, while $55 \%$ reported performing carbohydrate counting at least daily. A large proportion, $90.8 \%$ received prior T1D-specific education $(37.8 \%$ in the form of group education and the remainder as 1:1 teaching). Of those who have not attended T1D specific education, the reasons cited include not keen to attend (40\%), perceived lack of benefit (40\%) and lack of time (20\%).

\section{Assessment of hypoglycaemia and severe hypoglycaemia}

Of a total of 131 subjects, $22.5 \%$ had reported at least 1 episode of severe hypoglycaemia over the last one year. The prevalence of severe hypoglycaemia over the last one year in subjects with Clarke score $=<3$ and $>=4$ was $19.8 \%$ and $41.4 \%$ respectively, while in subjects with Gold score $=<3$ and $>=4$, the prevalence was $22.4 \%$ and $32.4 \%$ respectively. $19.1 \%$ had ever been previously hospitalized or attended emergency department for hypoglycaemia.

Eighty subjects (61.1\%) submitted SMBG records, of whom 53.8\% (43 subjects) had performed at least 3 blood glucose tests daily. During the 4 -week period of SMBG, the mean number of blood glucose readings per day was 3.1, while the mean blood glucose was $9.6 \mathrm{mmol} / \mathrm{L}$. Of the 80 subjects who submitted SMBG 
records, $38.8 \%$ had at least 1 episode of clinically significant hypoglycaemia (blood glucose $<3 \mathrm{mmol} / \mathrm{L}$ ) during the prospective 4 week period of SMBG.

\section{Assessment of IAH}

IAH was common. Of a total of 131 subjects, data for the Gold score was available for 120 subjects, while data for the Clarke score was available for 122 subjects. The remainder of the subjects had not completed the assessment for these scores.

Thirty-four subjects $(28.3 \%)$ had a Gold score $>=4$, while 29 subjects $(24.6 \%)$ had a Clarke score $>=4$. In the 120 subjects with both GOLD and Clarke scores available, $13.3 \%$ of subjects had IAH on both scores (Gold and Clarke scores both $>=4$ ), while $60.8 \%$ of subjects had intact awareness of hypoglycaemia on both scores (Gold and Clarke scores both $<=3$ ) (Table 2). When the Gold and Clarke scores were discordant, a higher proportion of subjects were identified to have IAH by a Gold score $>=4(15 \%, 18$ subjects $)$ than by a Clarke score $>=4(10.8 \%, 13$ subjects) (Table 2). There was moderate correlation between the Clarke and Gold scores $\left(r^{2}=0.415\right)$.

The Clarke score was associated with an increased frequency of hypoglycaemia during the prospective 4 week period of SMBG. The Clarke score was also associated with an increased frequency of severe hypoglycaemia over the last one year, as well as an increased frequency of ever being previously hospitalized or attending emergency department for hypoglycaemia (Table 3) .

The HYPO score was computed only for the subjects who performed at least 3 blood glucose tests daily. The median HYPO score was $6\left(90^{\text {th }}\right.$ percentile 80$)$. Significantly higher HYPO scores were seen in subjects with Clarke score $>=4$ compared to those with Clarke score $=<3$, although no significant difference was observed based on the GOLD score (Table 3).

\section{Association of IAH with self-management practices}

Adjustment of insulin doses for blood glucose using an insulin sensitivity factor (ISF) was associated with lower Gold and Clarke scores (Table 3). However, there was no significant association between adjustment of insulin doses for food and both scores. Prior attendance of diabetes group education, education from a dietitian and education on blood glucose targets were associated with lower Clarke scores, but not Gold scores (Table 4). There was no observed difference in Gold and Clarke scores with education from other healthcare professionals, such as a doctor or nurse. There was also no difference in both scores with prior education on SMBG, insulin injection technique, hypoglycaemia management, carbohydrate counting and sick day management.

\section{DISCUSSION}

Hypoglycaemia, severe hypoglycaemia and IAH are important clinical problems. Hypoglycaemia is the major limiting factor in achieving optimal glycaemic control ${ }^{2}$, while IAH is one of the most important risk factors for severe hypoglycaemia, increasing the risk up to six-fold ${ }^{5,9,14}$. Severe hypoglycaemia can lead to permanent cognitive impairment, cardiac arrhythmias, seizures and coma ${ }^{3,5,6,15,16}$. Other consequences of IAH include fear of hypoglycaemia, which leads to increased anxiety, decreased satisfaction and poor treatment compliance, reduced work productivity, strain in interpersonal relationships and decreased quality of life $\mathrm{e}^{5}$. To compound this problem, IAH is common in people with T1D. In prior studies, depending on the study population and method of assessment, the frequency of IAH can range from $19.5 \%$ to $62.5 \%$, 10,14,17. Therefore, assessment for both symptomatic and asymptomatic hypoglycaemia should be part of routine diabetes care in patients at risk for hypoglycaemia ${ }^{3,16}$. However, objective methods to screen for IAH are not in routine clinical use.

Based on Gold score $>=4$ and Clarke score $>=4$, the frequency of IAH in our study population was $28.3 \%$ and $24.6 \%$ respectively, which was similar to previous studies. In a study of 140 T1D subjects in Edinburgh by Geddes et al, using a Gold score of $>=4$ and Clarke score of $>=4$, the frequency of IAH was $24 \%$ and $26 \%$ respectively ${ }^{7}$. In a subsequent study by the same author of 518 subjects, $19.5 \%$ of subjects had IAH, 
based on a Gold score of $>=4^{14}$. A study by Choudhary et al found a similar IAH frequency of $22.1 \%$, based on a Gold score of $>=4^{15}$. That said, these studies were all done in Western populations - as far as we are aware, the Gold and Clarke scores have only been studied in one prior local study, which had looked at patients with Type 2 Diabetes (rather than T1D) ${ }^{18}$.

Despite fairly similar frequency of IAH, the frequency of severe hypoglycaemia was lower in our study $(32.4 \%$ and $41.4 \%$ in subjects with Gold score $>=4$ and Clarke score $>=4$ respectively) compared to the aforementioned studies by Geddes et al and Choudhary et al, in which the frequency of severe hypoglycaemia was 50.5 $-57.1 \%$ (in subjects with Gold score $>=4$ ) and $57 \%$ (in subjects with Clarke score $>=4)^{7,14,15}$. Increasing age and longer duration of diabetes are well-recognized risk factors for severe hypoglycaemia ${ }^{1,10,19-21}$. In our study, the subjects had a younger age (median age 35 years) and shorter duration of diabetes (14 years) compared to the subjects in these studies (mean age 45 - 52 years, mean duration of diabetes 23 34 years $)^{5,7,14,15}$, which might account for the observation of lower frequency of severe hypoglycaemia.

The Gold and Clarke scores are simple clinical tools which can be used to screen for IAH. The underlying basis of these scores is that T1D patients who believe they have reduced awareness of hypoglycaemia are generally correct ${ }^{8}$. Based on the literature, both Gold and Clarke scores have been validated to be associated with increased frequency of severe hypoglycaemia over the preceding one year and increased frequency of biochemical hypoglycaemia over a prospective 4 week period of $\mathrm{SMBG}^{5,7,8,14,15}$. The Clarke score also correlated with hypoglycaemic events on CGM, defined by glucose $<3.9 \mathrm{mmol} / \mathrm{L}$ and $<3 \mathrm{mmol} / \mathrm{L}^{22}$, which is not surprising as recurrent hypoglycaemic events leads to blunted counterregulatory hormonal responses $^{23,24}$. Both Gold and Clarke scores were associated with increasing age and longer duration of diabetes - aforementioned risk factors for severe hypoglycaemia ${ }^{5,7,14,15}$, and correlated well with each other $\left(\mathrm{r}^{2}=0.868\right)^{7,14}$.

In our study, IAH as defined by Clarke score $>=4$ was associated with increased frequency of hypoglycaemia during the prospective 4 week period of SMBG and increased frequency of severe hypoglycaemia over the preceding one year, which was consistent with the literature. However, no significant association between the Gold score and these outcomes was found in our study. There was also no observed association between both scores and age, duration of diabetes or glycaemic control. A positive, albeit weaker correlation $\left(\mathrm{r}^{2}=\right.$ 0.415) between the Gold and Clarke scores was observed.

There are several possibilities to explain the discrepancies between our study findings compared to the literature. Firstly, there is an inherent difference between the Gold and Clarke scores. The Clarke score involves eight questions while the Gold score requires just a single question. Therefore, the Gold score might be more susceptible to day-to-day variation, lower specificity and lower test-retest and inter-rater reliability ${ }^{25}$. When added to the fairly small sample size in our study, this might account for the lack of association between the Gold score and hypoglycaemic events, as well as the modest correlation with the Clarke score. Relevant to our findings, Hatle et al had found that the Clarke score was superior to the Gold score in identifying subjects at risk of severe hypoglycaemia and clinically significant hypoglycaemia ${ }^{25}$.

The HYPO score is a composite hypoglycaemia score designed by Ryan et al based on the frequency, severity and degree of unawareness of hypoglycaemia ${ }^{11}$. Its primary utility is to provide an objective discriminator of the severity of problems that occur with hypoglycaemia, in subjects who were being considered for islet transplantation ${ }^{11}$. Based on a cohort of T1D patients in Edmonton, a score of $<423$ indicated that hypoglycaemia was unlikely to be a major clinical concern, a score of 423 - 1046 indicated moderate problems with hypoglycaemia while a score of $>=1047$ (representing $90^{\text {th }}$ percentile) indicated severe problems with hypoglycaemia ${ }^{11}$. For comparison, in our study, the median HYPO score was 6 while the $90^{\text {th }}$ percentile score was 80 . However, the drawback of the HYPO score is that it is labour and resource intensive, requiring subjects to perform 4 weeks of SMBG followed by data collection and calculation of the scores, and is therefore less suitable for routine clinical use. We observed an association between the HYPO and Clarke score in our study - as far as we are aware, this is the first study to directly compare the utility of the HYPO and Clarke score. 
On the basis of the relatively high rates of IAH, serious consequences of severe hypoglycaemia and negligible cost of test administration, we propose the routine use of the Clarke score in all patients with T1D to identify $\mathrm{IAH}^{7}$. As described, the Clarke score is associated with the important clinical outcome of severe hypoglycaemia as well as diabetes self-management practices, which justifies its use despite its more time-consuming format. If there are limitations with its use, for instance due to time constraints in a busy outpatient clinic, we suggest that physicians obtain the Gold score despite its lower specificity ${ }^{25}$. As previously demonstrated by Pedersen et al, the distribution of severe hypoglycaemia is highly skewed, with $5 \%$ of subjects accounting for $54 \%$ of all episodes of severe hypoglycaemia ${ }^{17}$. Therefore, the benefit-risk ratio remains in favour of using these scores, which can allow the timely diagnosis of this subset of patients at high risk of severe hypoglycaemia and introduction of specific interventions to prevent serious complications.

For patients with IAH, the previous main recommendation was strict avoidance of hypoglycaemia by relaxation of glycaemic targets until restoration of glycaemic awareness ${ }^{3,9,26}$. However, this process might take several weeks to three months, lead to deterioration in glycaemic control and is difficult to achieve and sustain in clinical practice ${ }^{9,26}$. Thus, specific interventions can be taken to restore glycaemic awareness. These can broadly be divided into three groups: education, use of technology such as continuous glucose monitoring (CGM) and continuous subcutaneous insulin infusion (CSII), and pharmacological methods such as switching to insulin analogs ${ }^{9,24}$. For patients who are refractory to all other measures, islet cell transplantation can be considered ${ }^{24}$.

Our study confirms the central role of education in the potential reduction of IAH, which was previously demonstrated by the Dose Adjustment For Normal Eating-Hypoglycaemia Awareness Restoration Training (DAFNE-HART), HyPOS and HAATT trials ${ }^{27-30}$. These studies showed that in subjects with IAH, a psychobehavioural education program led to improved hypoglycaemic awareness with reductions in severe hypoglycaemia ${ }^{27-30}$.

In addition, our study clarified the content and delivery of education which is most efficacious. In our study, lower rates of IAH (based on the Clarke score) were seen in patients who had received group education, dietary education, education on self-adjustment of insulin doses and education on blood glucose targets. This is consistent with the findings by Yeoh et al that group education can improve hypoglycaemia awareness and reduce severe hypoglycaemia in up to $45 \%$ of people with $\mathrm{IAH}^{9}$. Thus, we suggest that T1D patients who have high Gold and/or Clarke scores be referred for structured diabetes education, in a group format if possible, to decrease the risk of IAH and its complications.

Our study is not without its limitations. This was a single centre study and the sample size is fairly small, which might account for the lack of association between the scores and established risk factors for IAH, as well as the modest correlation between the Gold and Clarke scores. In this study, capillary glucose testing was used, with about one-third of patients performing at least 3 blood glucose tests daily. At the time of this study, flash glucose monitoring was not available in Singapore, while continuous glucose monitoring was costly and not widely available. We acknowledge that episodes of hypoglycaemia may be missed during SMBG due to the limited frequency of testing. However, even today, capillary blood glucose testing (rather than continuous or flash glucose monitoring) remains the mainstay of home glucose monitoring for most of our patients. The continued utility of SMBG even today was well illustrated by the HypoCOMPaSS study, which showed that when subjects with IAH were provided with hypoglycaemia-focused structured education, glucose monitoring and optimised insulin replacement, there was no difference in hypoglycaemic awareness and treatment satisfaction between the different methods of glucose monitoring (SMBG vs real-time CGM) ${ }^{31}$.

Thus, as our study reflects the "real world" situation in actual clinical practice, we believe it remains relevant and provides important information for the management of T1D patients. In addition, the retrospective nature of the study in the form of a questionnaire precludes drawing conclusions about causation, for which an interventional study is required. Despite these limitations, we believe that based on this study, important recommendations which impact clinical practice can be drawn.

In conclusion, this is the first available study assessing IAH and its complications in a cohort of Singapore 
T1D subjects. Our study demonstrates that IAH is common and is associated with increased risk of serious complications, particularly severe hypoglycaemia. Simple questionnaires such as the Gold and Clarke scores should be routinely administered to identify patients with IAH who might benefit from specific interventions, particularly structured diabetes education, to decrease IAH and its complications.

\section{TABLES}

\section{Table 1: Baseline characteristics of subjects}

\begin{tabular}{ll}
\hline Characteristic & \\
\hline Age (years) & $35.9(17-69)$ \\
Age of diagnosis of diabetes (years) & $19(12-27)$ \\
Gender Male (\%) & 48 \\
Female (\%) & 52 \\
Race (\%) Chinese & 72.5 \\
Malays & 10.7 \\
Indian & 12.2 \\
Others & 4.6 \\
BMI (kg/m ${ }^{2}$ ) & $22.7(20.6-25.3)$ \\
Diabetes duration (years) & $14(8-19)$ \\
HbA1c (\%) & $8.2(7.3-9.2)$ \\
GAD antibody positive (\%) & 76.5 \\
Islet-cell antibody positive (\%) & 21.9 \\
Insulin regime Basal bolus insulin (\%) Premixed insulin $(\%)$ & 97.92 .1 \\
TDD of insulin (IU/kg) & $0.77(0.61-0.91)$ \\
Complications (\%) Retinopathy & 15.38 .44 .66 .93 .80 .80 .8 \\
Microalbuminuria & \\
Macroalbuminuria & \\
Neuropathy & \\
Ischemic heart disease & \\
Stroke & \\
Peripheral vascular disease & \\
\hline
\end{tabular}

Results are presented as median (interquartile range).

Table 2: Concordance between subjects with Gold and Clarke scores of $<=3$ and $>=4$

\begin{tabular}{llll}
\hline & $\begin{array}{l}\text { Subjects with Gold score } \\
<=3(\%)(\mathrm{N}=86)\end{array}$ & $\begin{array}{l}\text { Subjects with Gold score } \\
>=4(\%)(\mathrm{N}=34)\end{array}$ & P value \\
\hline $\begin{array}{l}\text { Subjects with Clarke } \\
\text { score }<=3(\%)(\mathrm{N}=91)\end{array}$ & $60.8(\mathrm{~N}=73)$ & $15(\mathrm{~N}=18)$ & $<0.001^{+}$ \\
$\begin{array}{l}\text { Subjects with Clarke } \\
\text { score }>=4(\%)(\mathrm{N}=\end{array}$ & $10.8(\mathrm{~N}=13)$ & $13.3(\mathrm{~N}=16)$ & \\
$29)$ & & & \\
\hline
\end{tabular}

\footnotetext{
+ Chi Square test
}

Total number of subjects: 120 (only subjects with available data for both the Gold and Clarke scores are included in this computation).

Table 3: Association between Gold and Clarke scores and a) Clinically significant hypogly- 
caemia during prospective 4 week period of SMBG b) Severe hypoglycaemia over the last one year c) Previous hospitalizations and emergency department attendances for hypoglycaemia d) HYPO score

\begin{tabular}{|c|c|c|c|c|c|c|}
\hline & $\begin{array}{l}\text { Subjects with } \\
\text { Gold score }<= \\
3(\mathrm{~N}=86)\end{array}$ & $\begin{array}{l}\text { Subjects with } \\
\text { Gold score }>= \\
4(\mathrm{~N}=34)\end{array}$ & $\mathrm{P}$ value & $\begin{array}{l}\text { Subjects with } \\
\text { Clarke score } \\
<=3(\mathrm{~N}=92)\end{array}$ & $\begin{array}{l}\text { Subjects with } \\
\text { Clarke score } \\
>=4(\mathrm{~N}=30)\end{array}$ & $\mathrm{P}$ value \\
\hline $\begin{array}{l}\text { Number of } \\
\text { hypogly- } \\
\text { caemic } \\
\text { episodes } \\
\text { [blood } \\
\text { glucose < } 3 \\
\text { mmol/L] } \\
\text { (median +/- } \\
\text { interquartile } \\
\text { range) } \\
\text { during } \\
\text { prospective } \\
4 \text { week } \\
\text { period of } \\
\text { SMBG }\end{array}$ & $\begin{array}{l}0.0(0.0- \\
1.0)\end{array}$ & $\begin{array}{l}0.0(0.0- \\
1.0)\end{array}$ & 0.408 & $\begin{array}{l}0.0(0.0- \\
1.0)\end{array}$ & $\begin{array}{l}1.0(0.0- \\
1.0)\end{array}$ & $0.006^{+}$ \\
\hline $\begin{array}{l}\text { Number of } \\
\text { episodes of } \\
\text { severe hypo- } \\
\text { glycaemia* } \\
\text { over the last } \\
\text { one year } \\
\text { (median +/- } \\
\text { interquartile } \\
\text { range) }\end{array}$ & $\begin{array}{l}0.0(0.0- \\
0.0)\end{array}$ & $\begin{array}{l}0.0(0.0- \\
1.0)\end{array}$ & 0.258 & $\begin{array}{l}0.0(0.0- \\
0.0)\end{array}$ & $\begin{array}{l}0.0(0.0- \\
1.0)\end{array}$ & $0.036^{+}$ \\
\hline $\begin{array}{l}\text { Number of } \\
\text { previous } \\
\text { hospitaliza- } \\
\text { tions and } \\
\text { emergency } \\
\text { department } \\
\text { attendances } \\
\text { for hypogly- } \\
\text { caemia } \\
\text { (median +/- } \\
\text { interquartile } \\
\text { range) }\end{array}$ & $\begin{array}{l}0.0(0.0- \\
0.0)\end{array}$ & $\begin{array}{l}0.0(0.0- \\
1.0)\end{array}$ & 0.093 & $\begin{array}{l}0.0(0.0- \\
0.0)\end{array}$ & $\begin{array}{l}0.0(0.0- \\
1.0)\end{array}$ & $0.010^{+}$ \\
\hline $\begin{array}{l}\text { HYPO score } \\
\text { (median +/- } \\
\text { interquartile } \\
\text { range) }\end{array}$ & $\begin{array}{l}0.0(0.0- \\
26.0)\end{array}$ & $\begin{array}{l}13.0(0.0- \\
68.75)\end{array}$ & 0.201 & $\begin{array}{l}0.0(0.0- \\
26.0)\end{array}$ & $\begin{array}{l}26.0(9.75- \\
81.0)\end{array}$ & $0.028^{+}$ \\
\hline
\end{tabular}

* Occurrence of at least 1 of the above events in the last one year: required assistance to recognize hypoglycaemia, required assistance to treat hypoglycaemia, required emergency medical services and required 
glucagon administration

+ Independent-samples median test

Number of subjects with available data for Gold score: 120

Number of subjects with available data for Clarke score: 122

Table 4: Association between Clarke score and a) diabetes self-management practices b) diabetes education

\begin{tabular}{llll}
\hline & $\begin{array}{l}\text { Subjects with Clarke } \\
\text { score }=<3(\%)(\mathrm{N}= \\
101)\end{array}$ & $\begin{array}{l}\text { Subjects with Clarke } \\
\text { score }>4(\%)(\mathrm{N}=30)\end{array}$ & P value \\
\hline $\begin{array}{l}\text { Subjects who adjust } \\
\text { insulin doses based on }\end{array}$ & 65.4 & 37.0 & $0.010^{+}$ \\
$\begin{array}{l}\text { ISF (\%) } \\
\begin{array}{l}\text { Subjects who received } \\
\text { education from }\end{array}\end{array}$ & 86.7 & 67.9 & $0.023^{+}$ \\
$\begin{array}{l}\text { dietitian (\%) } \\
\begin{array}{l}\text { Subjects who received } \\
\text { group education (\%) }\end{array}\end{array}$ & 43.8 & 21.4 & $0.034^{+}$ \\
$\begin{array}{l}\text { Subjects who received } \\
\text { education on blood } \\
\text { glucose targets (\%) }\end{array}$ & 87.6 & 65.4 & $0.008^{+}$ \\
\hline
\end{tabular}

+ Chi Square test

Number of subjects with available data for Clarke score: 122

\section{CONFLICTS OF INTEREST}

The authors declare that there is no conflict of interest.

\section{FUNDING STATEMENTS}

This work was supported by the Alexandra Health Enabling Grant. There are no other sources of funding or support. The funders had no role in study design, data collection and analysis, decision to publish, or preparation of the manuscript.

\section{ACKNOWLEDGEMENTS}

Not applicable.

\section{AUTHOR CONTRIBUTIONS}

All the authors have contributed to the preparation of this manuscript. A Koh conceptualized the study; E Yeoh, A Koh and S Fun contributed to data collection, study design and methodology; M Chua and A Koh performed data analysis; M Chua wrote the original draft of the manuscript; E Yeoh, A Koh and M Chua reviewed and edited the manuscript. In addition, all the authors are in agreement with the content of this manuscript.

\section{REFERENCES}

1. Diabetes Control and Complications Trial Research Group et al.The effect of intensive treatment of diabetes on the development and progression of long-term complications in insulin-dependent diabetes mellitus. N. Engl. J. Med. 329, 977-986 (1993). 
2. Seaquist, E. R. et al. Hypoglycemia and diabetes: a report of a workgroup of the American Diabetes Association and the Endocrine Society. Diabetes Care 36 , 1384-1395 (2013).

3. American Diabetes Association. 6. Glycemic Targets: Standards of Medical Care in Diabetes-2018. Diabetes Care 41 , S55-S64 (2018).

4. Graveling, A. J. \& Frier, B. M. Impaired awareness of hypoglycaemia: a review. Diabetes Metab. 36 Suppl 3, S64-74 (2010).

5. Martín-Timón, I. \& Del Cañizo-Gómez, F. J. Mechanisms of hypoglycemia unawareness and implications in diabetic patients. World J. Diabetes 6 , 912-926 (2015).

6. Gold, A. E., MacLeod, K. M. \& Frier, B. M. Frequency of severe hypoglycemia in patients with type I diabetes with impaired awareness of hypoglycemia. Diabetes Care 17, 697-703 (1994).

7. Geddes, J., Wright, R. J., Zammitt, N. N., Deary, I. J. \& Frier, B. M. An evaluation of methods of assessing impaired awareness of hypoglycemia in type 1 diabetes. Diabetes Care 30 , 1868-1870 (2007).

8. Clarke, W. L. et al. Reduced awareness of hypoglycemia in adults with IDDM. A prospective study of hypoglycemic frequency and associated symptoms. Diabetes Care 18, 517-522 (1995).

9. Yeoh, E., Choudhary, P., Nwokolo, M., Ayis, S. \& Amiel, S. A. Interventions That Restore Awareness of Hypoglycemia in Adults With Type 1 Diabetes: A Systematic Review and Meta-analysis. Diabetes Care 38 , 1592-1609 (2015).

10. Pedersen-Bjergaard, U., Agerholm-Larsen, B., Pramming, S., Hougaard, P. \& Thorsteinsson, B. Activity of angiotensin-converting enzyme and risk of severe hypoglycaemia in type 1 diabetes mellitus. Lancet Lond. Engl. 357 , 1248-1253 (2001).

11. Ryan, E. A. et al. Assessment of the severity of hypoglycemia and glycemic lability in type 1 diabetic subjects undergoing islet transplantation. Diabetes 53 , 955-962 (2004).

12. Pedersen-Bjergaard, U., Pramming, S. \& Thorsteinsson, B. Recall of severe hypoglycaemia and selfestimated state of awareness in type 1 diabetes. Diabetes Metab. Res. Rev. 19 , 232-240 (2003).

13. International Hypoglycaemia Study Group. Glucose Concentrations of Less Than $3.0 \mathrm{mmol} / \mathrm{L}$ (54 mg/dL) Should Be Reported in Clinical Trials: A Joint Position Statement of the American Diabetes Association and the European Association for the Study of Diabetes. Diabetes Care40 , 155-157 (2017).

14. Geddes, J., Schopman, J. E., Zammitt, N. N. \& Frier, B. M. Prevalence of impaired awareness of hypoglycaemia in adults with Type 1 diabetes. Diabet. Med. J. Br. Diabet. Assoc. 25 , 501-504 (2008).

15. Choudhary, P. et al. Frequency of biochemical hypoglycaemia in adults with Type 1 diabetes with and without impaired awareness of hypoglycaemia: no identifiable differences using continuous glucose monitoring. Diabet. Med. J. Br. Diabet. Assoc. 27 , 666-672 (2010).

16. Kahkoska, A. R. \& Buse, J. B. Primum Non Nocere: Refocusing Our Attention on Severe Hypoglycemia Prevention. Diabetes Care41, 1557-1559 (2018).

17. Pedersen-Bjergaard, U. et al. Severe hypoglycaemia in 1076 adult patients with type 1 diabetes: influence of risk markers and selection. Diabetes Metab. Res. Rev. 20 , 479-486 (2004).

18. Zhu, L. et al. A study to evaluate the prevalence of impaired awareness of hypoglycaemia in adults with type 2 diabetes in outpatient clinic in a tertiary care centre in Singapore. Ther. Adv. Endocrinol. Metab. 8 , 69-74 (2017).

19. Pedersen-Bjergaard, U. et al. Severe hypoglycaemia in 1076 adult patients with type 1 diabetes: influence of risk markers and selection. Diabetes Metab. Res. Rev. 20 , 479-486 (2004). 
20. Lee, A. K. et al. Risk Factors for Severe Hypoglycemia in Black and White Adults With Diabetes: The Atherosclerosis Risk in Communities (ARIC) Study. Diabetes Care 40 , 1661-1667 (2017).

21. Matsuhisa, M. \& Kuroda, A. New risk factors of severe hypoglycemia.J. Diabetes Investig. 10 , 219-220 (2019).

22. Lin, Y. K. et al. Associations Between the Time in Hypoglycemia and Hypoglycemia Awareness Status in Type 1 Diabetes Patients Using Continuous Glucose Monitoring Systems. Diabetes Technol. Ther. 22 , 787-793 (2020).

23. Cryer, P. E. Mechanisms of hypoglycemia-associated autonomic failure in diabetes. N. Engl. J. Med. 369 , 362-372 (2013).

24. Choudhary, P. et al. Evidence-informed clinical practice recommendations for treatment of type 1 diabetes complicated by problematic hypoglycemia. Diabetes Care 38 , 1016-1029 (2015).

25. Hatle, H. et al. Assessing awareness of hypoglycemia in children and adolescents with type 1 diabetes: Evaluation of established questionnaires. Pediatr. Diabetes 21 , 300-309 (2020).

26. Diabetes Canada Clinical Practice Guidelines Expert Committee, Yale, J.-F., Paty, B. \& Senior, P. A. Hypoglycemia. Can. J. Diabetes42 Suppl 1, S104-S108 (2018).

27. Hermanns, N., Kulzer, B., Kubiak, T., Krichbaum, M. \& Haak, T. The effect of an education programme (HyPOS) to treat hypoglycaemia problems in patients with type 1 diabetes. Diabetes Metab. Res. Rev.23, $528-538$ (2007).

28. Hermanns, N., Kulzer, B., Krichbaum, M., Kubiak, T. \& Haak, T. Long-term effect of an education program (HyPOS) on the incidence of severe hypoglycemia in patients with type 1 diabetes. Diabetes Care 33, e36 (2010).

29. Cox, D. J. et al. Hypoglycemia anticipation, awareness and treatment training (HAATT) reduces occurrence of severe hypoglycemia among adults with type 1 diabetes mellitus. Int. J. Behav. Med.11, 212-218 (2004).

30. de Zoysa, N. et al. A psychoeducational program to restore hypoglycemia awareness: the DAFNE-HART pilot study. Diabetes Care37 , 863-866 (2014).

31. Little, S. A. et al. Recovery of hypoglycemia awareness in long-standing type 1 diabetes: a multicenter $2 \times$ 2 factorial randomized controlled trial comparing insulin pump with multiple daily injections and continuous with conventional glucose self-monitoring (HypoCOMPaSS).Diabetes Care 37 , 2114-2122 (2014). 